ARTICLE

\title{
Demonstration of chip-based coupled degenerate optical parametric oscillators for realizing a nanophotonic spin-glass
}

\author{
Yoshitomo Okawachi (i) 1,4, Mengjie Yu1,2,4, Jae K. Jang ${ }^{1}$, Xingchen Ji id ${ }^{3}$, Yun Zhao ${ }^{3}$, Bok Young Kim (i) ${ }^{1}$, \\ Michal Lipson (1) ${ }^{1,3} \&$ Alexander L. Gaeta (1) ${ }^{1,3 \times}$
}

The need for solving optimization problems is prevalent in various physical applications, including neuroscience, network design, biological systems, socio-economics, and chemical reactions. Many of these are classified as non-deterministic polynomial-time hard and thus become intractable to solve as the system scales to a large number of elements. Recent research advances in photonics have sparked interest in using a network of coupled degenerate optical parametric oscillators (DOPOs) to effectively find the ground state of the Ising Hamiltonian, which can be used to solve other combinatorial optimization problems through polynomial-time mapping. Here, using the nanophotonic silicon-nitride platform, we demonstrate a spatial-multiplexed DOPO system using continuous-wave pumping. We experimentally demonstrate the generation and coupling of two microresonator-based DOPOs on a single chip. Through a reconfigurable phase link, we achieve both in-phase and out-of-phase operation, which can be deterministically achieved at a fast regeneration speed of $400 \mathrm{kHz}$ with a large phase tolerance.

\footnotetext{
${ }^{1}$ Department of Applied Physics and Applied Mathematics, Columbia University, New York, NY 10027, USA. ${ }^{2}$ School of Electrical and Computer Engineering, Cornell University, Ithaca, NY 14853, USA. ${ }^{3}$ Department of Electrical Engineering, Columbia University, New York, NY 10027, USA. ${ }^{4}$ These authors contributed equally: Yoshitomo Okawachi and Mengjie Yu. ${ }^{凶}$ email: a.gaeta@columbia.edu
} 
T he processing speed of modern computers is limited by the fact that program memory and data memory share the same bus. While processors have become faster, the overall speed is limited by the data transfer rate, known as the von Neumann bottleneck ${ }^{1}$. There has been an increase in demand for solving certain classes of computation problems that scale exponentially in time and energy for the current von Neumann architecture. Many combinatorial optimization problems fall under this category and are classified as non-deterministic polynomial-time (NP) hard and have applications in areas including finance, scheduling, trajectory planning, and artificial intelligence. The ability to solve these problems efficiently has motivated the development of computing accelerators based on digital and physical systems ${ }^{2-29}$.

Recently, there has been considerable interest in using photonic processors to realize a novel form of coherent computing by simulating the Ising model ${ }^{16,20-22}$. The Ising model was developed for modeling ferromagnetism and is governed by a Hamiltonian that couples discrete variables that represent spin glasses $^{30,31}$. Solving for the ground state of such a system corresponds to solving an NP-hard computational problem and can provide an architecture for solving other NP-complete problems through polynomial-time mapping to the Ising model ${ }^{32}$. The Ising Hamiltonian with $N$ spins and no external field is given by $H=-\sum_{i j}^{N} J_{i j} \sigma_{i} \sigma_{j}$, where $J_{i j}$ is the coupling coefficient and $\sigma_{i}$ corresponds to the projection of the $i$ th spin along the $z$-axis that can have two states \pm 1 . The physical realization of an Ising machine requires binary degrees of freedom (i.e., spins $\sigma_{i}$ ) and reconfigurable coupling (i.e., $J_{i j}$ ), and initially was studied using a network of injection-locked lasers ${ }^{10-15}$. More recently, investigations have shown that a network of coupled degenerate optical parametric oscillators (DOPOs) based on the $\chi^{(2)}$ nonlinearity can be used to realize a hybrid temporally multiplexed coherent Ising machine ${ }^{16-21}$, which includes a recent demonstration of a system of 2000 spins $^{20}$. The nonlinearity is based on the nonequilibrium phase transition that occurs at the parametric oscillation threshold, resulting in two possible phase states of the DOPO offset by $\pi$, and the couplings between the DOPOs are implemented via measurement feedback or optical delay lines. By controlling the coupling between these DOPOs, it is possible to achieve more complex, phase-locked output states that encode the ground state of an Ising model. These demonstrations have utilized a time-multiplexed DOPO system using a 1-km-long fiber ring cavity to simulate the ground state of the Ising model ${ }^{20,21}$. In addition, extensive experimental and theoretical analysis has been done to characterize the potential performance of such systems $^{23,33-35}$. Furthermore, alternative approaches towards a coherent Ising machine, such as opto-electronic oscillators with self-feedback ${ }^{24-26}$ and spatial light modulation ${ }^{27,28}$, has been demonstrated and an approach using a dispersive optical bistability has been proposed ${ }^{29}$.

An alternative approach to realize a DOPO is to use the $\chi^{(3)}$ nonlinearity in which a frequency degenerate signal/idler pair is generated via parametric four-wave mixing $(\mathrm{FWM})^{36-39}$. Such a scheme has been implemented using silicon nitride $\left(\mathrm{SiN}, \mathrm{Si}_{3} \mathrm{~N}_{4}\right)$ microresonators and bi-phase state generation has been achieved enabling quantum random-number generation in a chip-scale device (Fig. 1) ${ }^{37,39}$. The SiN platform is ideally suited for scalability to an all-photonic network of coupled DOPOs since it is CMOS (complementary metal-oxide-semiconductor) process compatible, has low losses in the near infrared, and allows for dispersion engineering, which is crucial for efficient phase-matched nonlinear processes ${ }^{40}$. Unlike the $\chi^{(2)}$ process, the wavelengths of the pump and degenerate signal are spectrally close, allowing for phase matching of the signal through

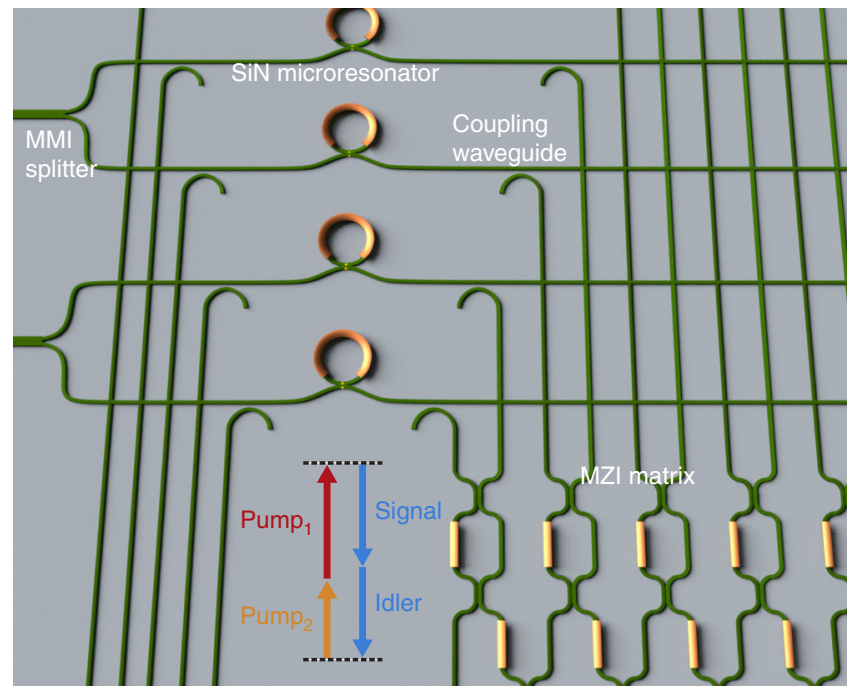

Fig. 1 Conceptual schematic of a network of coupled degenerate optical parametric oscillators. Two-frequency nondegenerate pumps are injected into the network of coupled degenerate optical parametric oscillators (DOPOs) based on silicon nitride ( $\mathrm{SiN}$ ) microresonators. The pumps are split on-chip using multimode interference (MMI) splitters. The DOPO signal is phase matched and generated via four-wave mixing parametric oscillation. Phase-matching conditions result in a bi-phase state for the generated signal. Coupling between DOPOs can be performed using a matrix of reconfigurable Mach-Zehnder interferometers (MZIs).

dispersion engineering of the fundamental waveguide mode. In addition, unlike traditional FWM, which requires operation in the anomalous group-velocity dispersion (GVD) regime, the DOPO requires normal GVD for phase matching 37 , which is more readily accessible across a wider range of photonic platforms. Furthermore, the microresonator-based DOPO system allows for simultaneous oscillation of all DOPOs, enables continuous-wave (cw) operation, and does not rely on long cavity lengths as in the time-multiplexing scheme, which requires phase stabilization to support multiple trains of femtosecond pulses, offering faster computational speeds with lower power consumption in a compact footprint. A recent preliminary study has also reported on numerical simulation of coupled Lugiato-Lefever equations to tackle the MAX-CUT problem, further demonstrating the potential capability of SiN platform for optical computing ${ }^{41}$.

In this paper, we demonstrate an integrated photonic circuit that consists of spatial-multiplexed DOPOs on a single SiN chip and present the first demonstration of a coupled DOPO system using $\mathrm{cw}$ pumping. We experimentally show reconfigurability of the coupling phase between the two DOPOs through thermal control of the coupling waveguide between the DOPOs and show interference measurements between the DOPOs indicating inphase and out-of-phase operation. In our system, we solve at a $400-\mathrm{kHz}$ rate with a convergence time of $<310 \mathrm{~ns}$. In addition, we numerically model the coupled DOPO system and confirm the behavior for in-phase and out-of-phase operation and explore the transition region between the two phase states. A distinct transition region between two states is revealed both numerically and experimentally, suggesting a tremendous phase tolerance of such parametric process. We also theoretically investigate our coupled DOPO system using coupled Lugiato-Lefever equations and describe the scalability of such a system to a large number of oscillators on-chip and the challenges in achieving a large-scale photonic coherent Ising machine. 
a

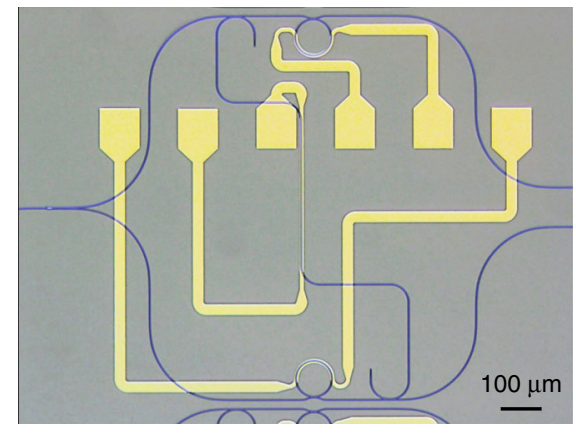

b

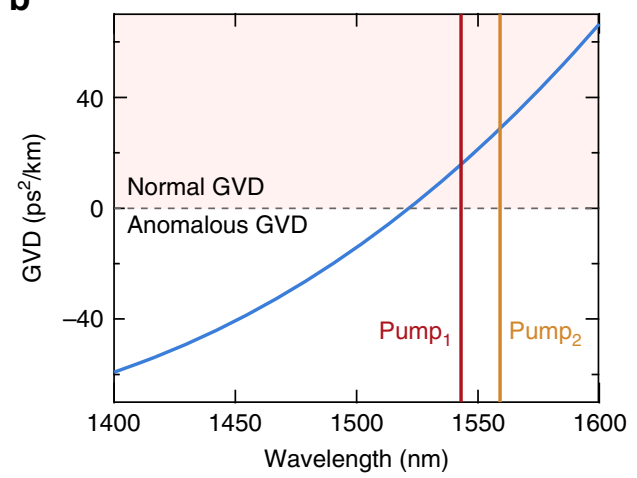

C

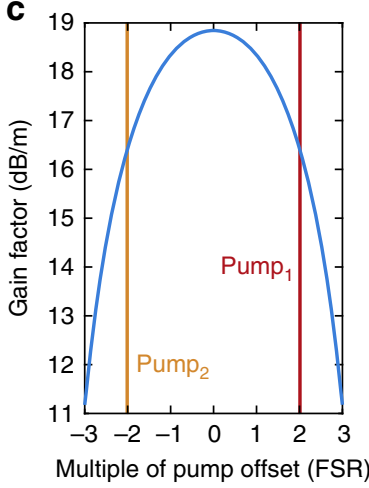

d

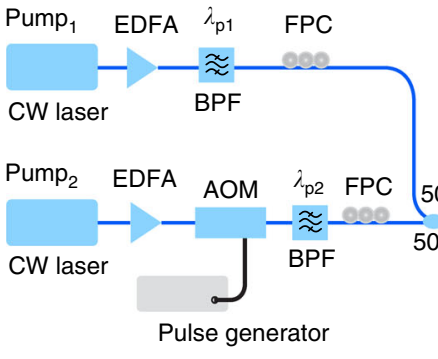

Pulse generator

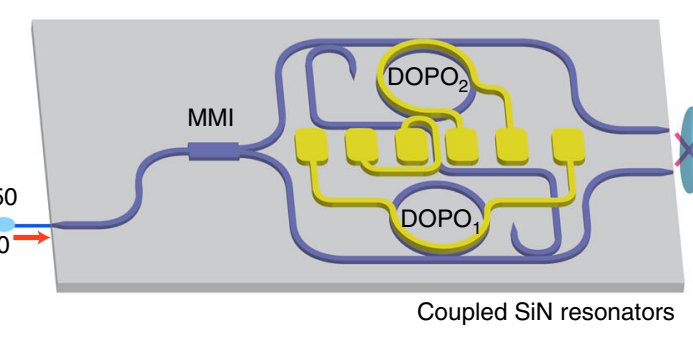

Coupled SiN resonators

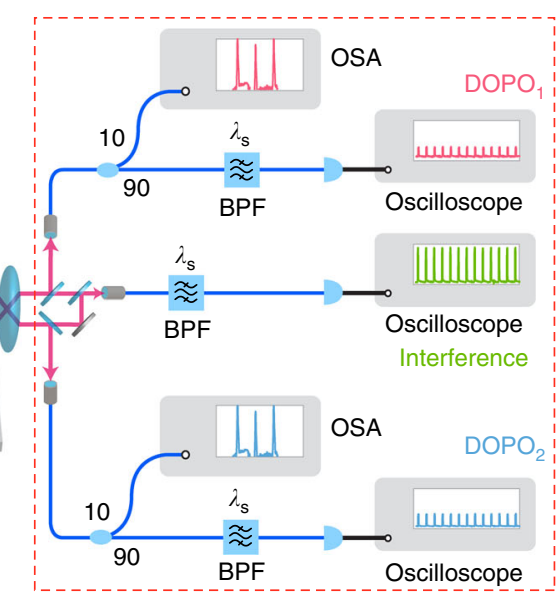

Fig. 2 Experimental schematic of coupled DOPOs. a Microscope image of the device. The pump waves are split on-chip and sent to $D_{0 P O_{1}}($ bottom) and $\mathrm{DOPO}_{2}$ (top). A coupling waveguide after $\mathrm{DOPO}_{1}$ is used to send a fraction of the $\mathrm{DOPO}_{1}$ field to $\mathrm{DOPO}_{2}$. $\mathbf{b}$ Simulated group-velocity dispersion (GVD) of the $\mathrm{SiN}$ microresonator for the fundamental transverse electric (TE) mode. The waveguide cross section is $730 \times 1050 \mathrm{~nm}^{2}$. The region of normal GVD is shaded, and the pump wavelengths are indicated with vertical lines. c Calculated parametric gain for $1 \mathrm{~W}$ of combined pump power. The pump waves are each located two free spectral ranges (FSRs) from the degeneracy point. $\mathbf{d}$ Experimental setup for measurement of coupled DOPO system. Two-frequency nondegenerate pumps are sent into the SiN chip. The output is collected using an aspheric lens and sent to a free-space interferometer to measure the interference signal. EDFA erbium-doped fiber amplifier, BPF bandpass filter, AOM acousto-optic modulator, FPC fiber polarization controller, MMI multimode interference splitter, OSA optical spectrum analyzer.

\section{Results}

Device characterization and experimental setup. The key components for realizing a network of coupled DOPOs on-chip are, (1) power splitters for routing the pump fields to the microresonators, (2) nonlinear microresonators designed for DOPO generation, and (3) the $N \times N$ photonic coupling system between the different microresonators. A microscope image of the SiN device is shown in Fig. $2 \mathrm{a}$ and is fabricated using techniques similar to those reported in Luke et al. ${ }^{42}$. For routing the twofrequency nondegenerate pumps to the microresonators, we employ an on-chip power splitter using multimode interference $(\mathrm{MMI})^{43}$, where the dimensions of the MMI are designed to allow for 50/50 power splitting ratio. The insertion loss of the MMI splitter is $2 \mathrm{~dB}$. The two $\mathrm{SiN}$ microresonators $\left(\mathrm{DOPO}_{1}\right.$ and $\mathrm{DOPO}_{2}$ ) have a radius of $45.84 \mu \mathrm{m}$, which corresponds to a free spectral range (FSR) of $500 \mathrm{GHz}$. The loaded quality $(Q)$ factors of the microresonators are 630,000. The condition $L_{\mathrm{D}}>L_{\mathrm{NL}}$, where $L_{\mathrm{D}}=1 / \delta^{2}\left|\beta_{2}\right|$ is the dispersion length and $L_{\mathrm{NL}}=1 / 2 \gamma P$ is the nonlinear length, is critical for achieving maximum gain at the frequency degeneracy point and enabling pure DOPO generation (see Supplementary Note 1$)^{37}$. Here, $\beta_{2}$ is the GVD parameter, $\gamma$ is the nonlinear parameter, $P$ is the power of each pump, and $\delta$ is the pump frequency offset. Based on simulations using a finite-element mode solver, we use a waveguide cross section of $730 \times 1050 \mathrm{~nm}^{2}$ such that the two pumps are placed in the normal GVD regime for the fundamental transverse electric (TE) mode to allow for efficient phase matching and maximum gain at the frequency degeneracy point ${ }^{37}$. The simulated GVD is shown in Fig. 2b, and the corresponding gain for $1 \mathrm{~W}$ of pump power is shown in Fig. 2c. The cavity resonance for each microresonator is thermally controlled using integrated platinum resistive microheaters. The coupling between the microresonator and the bus waveguide is designed to have near-critical coupling, where the extinction ratio of the resonances is $\sim 92 \%$. To compensate for the difference in the resonance frequencies of the two resonators due to the fabrication tolerances in microresonator geometry, we use microheaters above each resonator that allow for electrical control of the resonances via the thermo-optic effect $^{44,45}$. We set the electrical power to the heaters such that the cavity resonances for the two microresonators corresponding to both pump frequencies overlap. We implement reconfigurable unidirectional coupling between the two DOPOs by using a coupling waveguide that directs a fraction of the $\mathrm{DOPO}_{1}$ output field to the input of $\mathrm{DOPO}_{2}$. The coupling strength of the coupling waveguide is adjusted by designing the separation between the bus waveguide and coupling waveguide. In our device, the ratio between the coupled field from $\mathrm{DOPO}_{1}$ and the $\mathrm{DOPO}_{2}$ field is 0.048 (see Supplementary Note 2). In order to minimize reflections, we have implemented a taper design on the end facets. We tune the phase coupling between the two DOPOs 
from in-phase to out-of-phase by thermally tuning the path length using microheaters.

Figure $2 \mathrm{~d}$ shows the experimental setup for generation and detection of the coupled DOPO signal. The SiN chip is pumped using two-frequency nondegenerate $\mathrm{cw}$ pump lasers that are offset by \pm 2 FSRs from the degeneracy point at wavelengths of 1543 and $1559.1 \mathrm{~nm}$. In order to ensure that the DOPO builds up from noise for each initiation, we modulate one of the pumps using an acousto-optic modulator (AOM) with 310-ns pulses at a repetition rate of $400 \mathrm{kHz}$. For simultaneous degenerate oscillation for both DOPOs, we use $72 \mathrm{~mW}$ of combined pump power in each bus waveguide and set the electrical power to the heater in $\mathrm{DOPO}_{1}$ to $13.5 \mathrm{~mW}$ such that the resonances of the two microresonators are spectrally overlapped. The two DOPO outputs from the chip are collimated using an aspheric lens and sent to our detection setup [dashed red box in Fig. 2a].

Phase characterization of coupled DOPOs. The readout of the coherent phase states of the coupled DOPO system is implemented by directly measuring the interference between the two DOPOs by coupling both outputs into a single fiber collimator and detecting the combined signal on a fast photodiode. The collimated outputs from the DOPOs are combined using a 50/50 beamsplitter and fiber coupled using a collimator. Before combining the beams, a 50/50 beamsplitter is used in each output arm to collect the individual DOPO signals. The signals are each fiber coupled and a 90/10 coupler is used to monitor the time trace and the optical spectrum. The generated DOPO spectra from the microresonators are shown in Fig. 3. In our interference measurement, we manipulate the coupling phase $\phi_{c}$ between the DOPOs below threshold by controlling the electrical power sent to the integrated heater while monitoring the time trace. Figure 4 shows the measured temporal interference signal (green) along with $\mathrm{DOPO}_{1}$ (red) and $\mathrm{DOPO}_{2}$ (blue), for three different heater powers. At $48.3 \mathrm{~mW}$, we observe constructive interference between the two DOPOs, corresponding to in-phase operation $\left(\phi_{\text {out }}=0\right)$ [Fig. 4 (top)]. For $54.2 \mathrm{~mW}$ of heater power, we observe destructive interference [Fig. 4 (middle)] corresponding to out-of-phase operation $\left(\phi_{\text {out }}=\pi\right)$. The transition from inphase to out-of-phase operation occurs at $50.2 \mathrm{~mW}$ of heater power, and we observe both constructive and destructive interference [Fig. 4 (bottom)]. Moreover, we observe in-phase operation $\left(\phi_{\text {out }}=0\right)$ for heater powers below $48.3 \mathrm{~mW}$ and out-of-phase operation $\left(\phi_{\text {out }}=\pi\right)$ for powers above $54.2 \mathrm{~mW}$, indicating that the DOPOs above threshold operate in-phase or out-of-phase for a continuous range of coupling phases $\phi_{c}$. The convergence time of the DOPO is well within the pump pulse duration of $310 \mathrm{~ns}$. For comparison, we perform interference measurements on a similar device with no coupling waveguide between $\mathrm{DOPO}_{1}$ and $\mathrm{DOPO}_{2}$ and verify that there is no phase correlation between the two signals (see Supplementary Note 3).

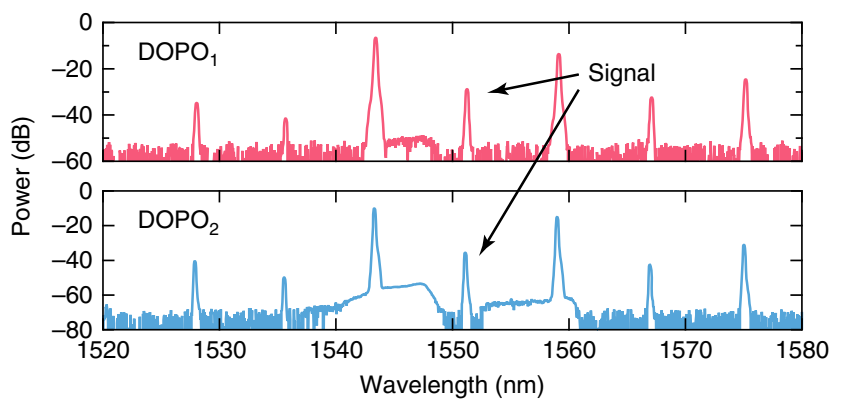

Fig. 3 Optical spectra of two DOPO signals. The measured spectra of the primary DOPO (top, DOPO$)$ and the secondary DOPO (bottom, $\mathrm{DOPO}_{2}$ ).
Theoretical analysis of coupled DOPO system. We theoretically investigate this coupled DOPO system using coupled Lugiato-Lefever equations with two pump waves ${ }^{41,46}$,

$$
\begin{aligned}
t_{\mathrm{R}} \frac{\partial E_{1}}{\partial t}= & {\left[-\alpha-i \delta_{0}-\delta_{1} \frac{\partial}{\partial \tau}+i L \sum_{k \geq 2} \frac{\beta_{k}}{k !}\left(i \frac{\partial}{\partial \tau}\right)^{k}+i \gamma L\left|E_{1}(t, \tau)\right|^{2}\right] E_{1}(t, \tau) } \\
& +\sqrt{\theta} A_{\text {in }}\left(e^{-i \Omega_{0} \tau}+e^{i \Omega_{0} \tau}\right),
\end{aligned}
$$

$$
\begin{aligned}
t_{\mathrm{R}} \frac{\partial E_{2}}{\partial t}= & {\left[-\alpha-i \delta_{0}-\delta_{1} \frac{\partial}{\partial \tau}+i L \sum_{k \geq 2} \frac{\beta_{k}}{k !}\left(i \frac{\partial}{\partial \tau}\right)^{k}+i \gamma L\left|E_{2}(t, \tau)\right|^{2}\right] E_{2}(t, \tau) } \\
& +\sqrt{\theta} A_{\mathrm{in}}\left(e^{-i \Omega_{0} \tau}+e^{i \Omega_{0} \tau}\right)+\kappa E_{1}(t, \tau),
\end{aligned}
$$

where $t_{\mathrm{R}}$ is the roundtrip time in the resonator, $\alpha$ is the total roundtrip loss, $\delta_{0}$ is the effective phase detuning, $\delta_{1}$ is the modedependent detuning, $\Omega_{0}$ corresponds to the pump offset frequency from the degeneracy point, $\theta$ is the transmission coefficient between the resonator and the bus waveguide, $L$ is the cavity length, $\gamma$ is the nonlinear parameter, and $\beta_{k}$ corresponds to the $k$ th-order dispersion coefficients of the Taylor expansion of the propagation constant. Here, $\tau$ represents the temporal coordinate within the time scale of a single round trip and $t$ represents the long-time-scale evolution over many round trips. The term with $A_{\text {in }}$ describes the bichromatic pump waves, and $\kappa$ represents the complex coupling coefficient from the primary DOPO $\left(\mathrm{DOPO}_{1}\right)$ to the secondary DOPO $\left(\mathrm{DOPO}_{2}\right)$ (see Supplementary Note 4 ). We add a noise of one photon per spectral mode with random phase onto the pump ${ }^{47}$. The simulation parameters are similar to that of our experiment, including the coupling between the DOPOs being unidirectional. Our simulations (Fig. 5) show oscillation behavior for three different values of the coupling phase $\phi_{c}$. For $\phi_{c}=0^{\circ}$, constructive interference between the DOPOs is favored [Fig. 5 (top)], indicating that the DOPOs oscillate in-phase. We observe similar in-phase behavior for $-70^{\circ}<\phi_{\mathrm{c}}<70^{\circ}$. In contrast, for $\phi_{\mathrm{c}}=180^{\circ}$, we observe destructive interference [Fig. 5 (middle)] from the DOPOs oscillating $\pi$ outof-phase. Likewise, similar out-of-phase behavior is observed for $110^{\circ}<\phi_{\mathrm{c}}<250^{\circ}$. These predictions are consistent with our experimental results. In addition, we have numerically explored the transition region between in-phase and out-of-phase operation. Figure 5 (bottom) shows the simulated interference for $\phi_{c}=90^{\circ}$. Here, we observe that the secondary DOPO no longer oscillates out-of-phase with respect to the primary DOPO and becomes frustrated with the oscillation becoming uncorrelated, which is also consistent with our experimental observations for the transition region (heater power of $50.2 \mathrm{~mW}$ ). Work is ongoing to determine the degree of phase tolerance as the number of DOPOs is increased.

Scalability of coupled DOPO system. Lastly, we discuss the potential of a large-scale DOPO photonic system in light of power consumption and computing speed. Figure 6a shows the combined pump power required for a single microresonator-based DOPO to oscillate as a function of the intrinsic $Q$-factor of the $\mathrm{SiN}$ microresonator for critical coupling. The measured values for the current device and a similar single DOPO device are denoted with a diamond (see Supplementary Note 5). For a $Q$-factor of 10 million, oscillation can occur with $1 \mathrm{~mW}$ of combined pump power, implying that 1000 DOPOs can be pumped simultaneously with an on-chip optical power of $1 \mathrm{~W}$, offering promise for scaling to a large number of DOPOs (see Supplementary Note 6). Here, since the power scales as $1 / Q^{2}$ and the lifetime scales as $Q$, the energy required for the entire computation scales as $1 / Q$. With reduction of surface roughness, $Q$-factors of 37 

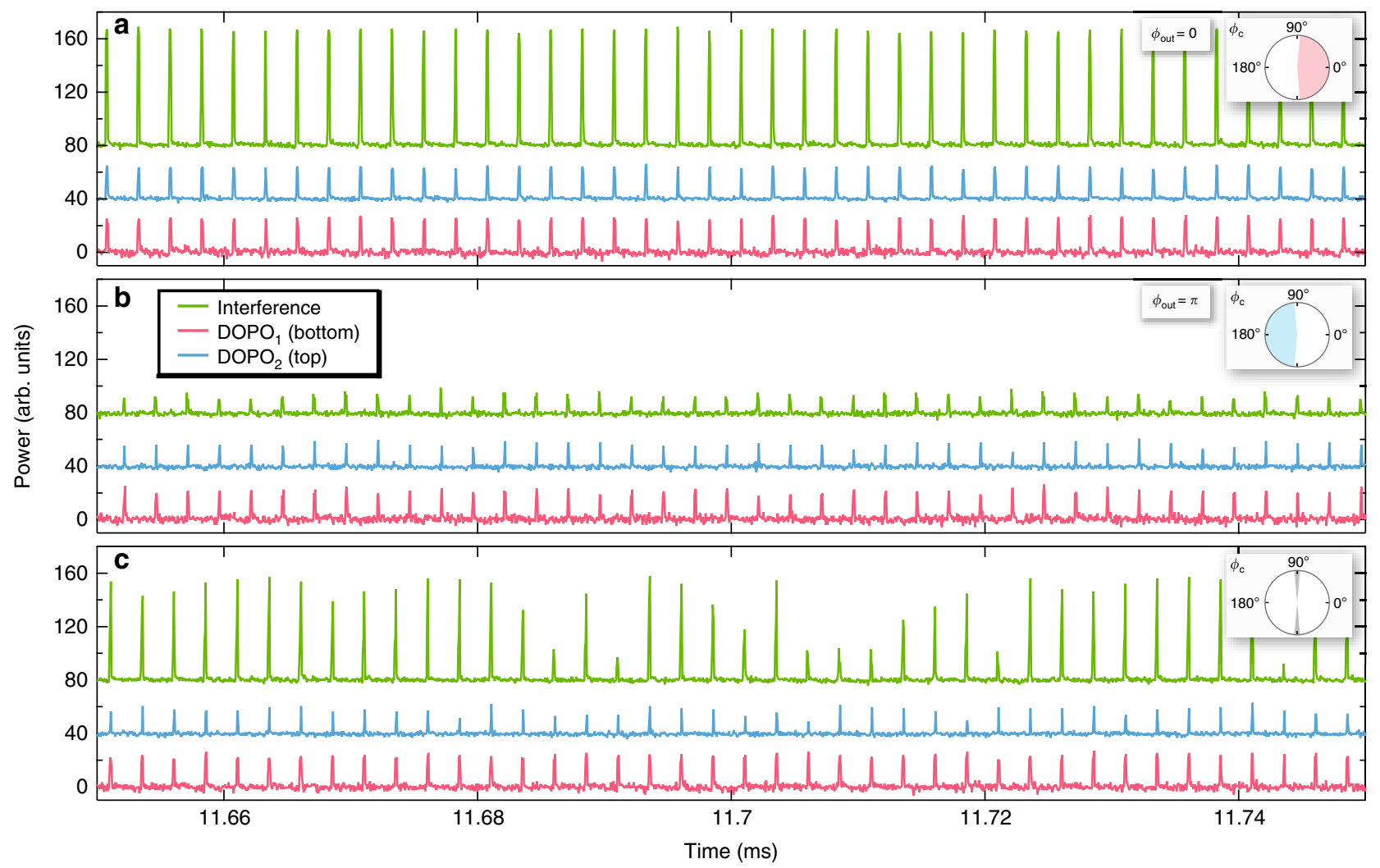

Fig. 4 Phase characterization of coupled DOPOs. Temporal ensemble of interference measurements of the combined DOPOs (green) along with measurements of $\mathrm{DOPO}_{1}$ (red) and $\mathrm{DOPO}_{2}$ (blue). Inset shows the phase chart of the coupling phase $\phi_{\mathrm{c}}$. We indicate the coupled DOPO operation regime for in-phase (pink), out-of-phase (blue), and transition regime (gray). a Constructive interference (in-phase, $\phi_{\text {out }}=0$ ) is observed for a heater power of $48.3 \mathrm{~mW}$. b Destructive interference (out-of-phase $\phi_{\text {out }}=\pi$ ) is observed for a heater power of $54.2 \mathrm{~mW}$. c Transition from in-phase to out-of-phase operation for heater power of $50.2 \mathrm{~mW}$. For clarity, $\mathrm{DOPO}_{2}$ and the interference are offset from $\mathrm{DOPO}_{1}$ in power by 40 and 80 , respectively.

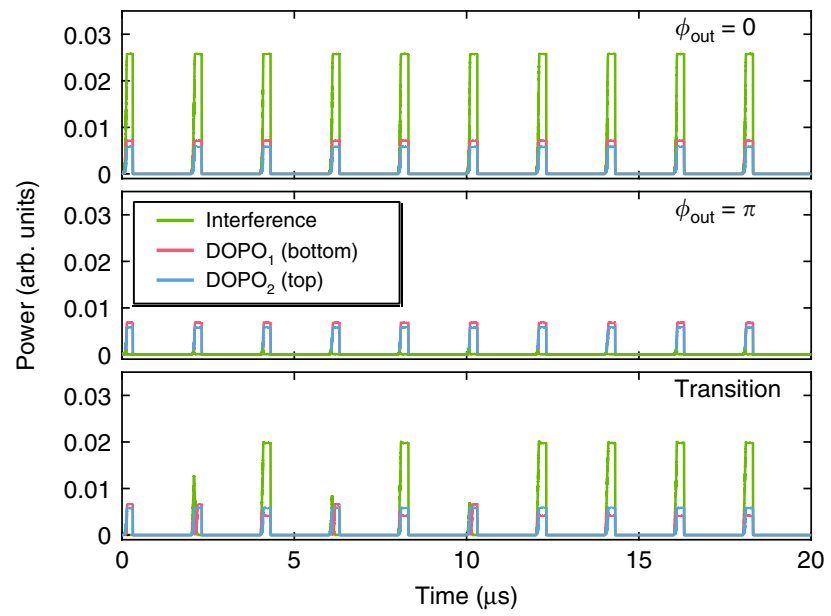

Fig. 5 Numerical modeling of coupled DOPO system. Simulated temporal interference between two DOPOs with unidirectional coupling. The plot shows the combined DOPO (green) and the individual DOPOs, $\mathrm{DOPO}_{1}$ (red) and $\mathrm{DOPO}_{2}$ (blue) for coupling phase $\phi_{\mathrm{c}}$ of $0^{\circ}$ (in-phase, $\phi_{\text {out }}=0$ ), $180^{\circ}$ (out-of-phase, $\phi_{\text {out }}=\pi$ ), and $90^{\circ}$ (transition from in-phase to out-ofphase) from top to bottom.

million have been achieved in high-confinement SiN microresonators ${ }^{48}$, which could further reduce the pump power to 80 $\mu \mathrm{W}$ per DOPO (labeled with a triangle in Fig. 6a), at the expense of computing rate. In addition, Fig. $6 \mathrm{~b}, \mathrm{c}$ shows the simulated DOPO oscillation time for two different $Q$-factors at near-
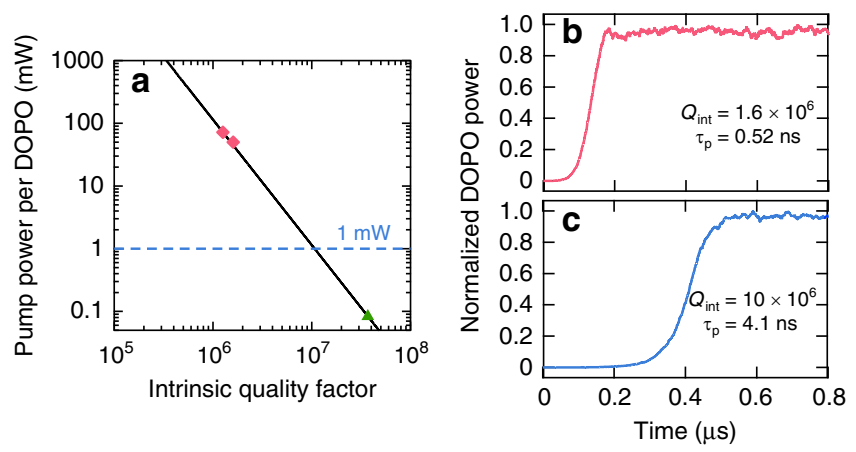

Fig. 6 Scaling to larger number of coupled DOPOs. a Combined pump power required for single DOPO as a function of the intrinsic $Q$-factor of the microresonator. The two different pump powers (and the corresponding $Q$ ) used in our experiments is denoted with diamonds. Triangle (green) denotes pump power based on the $Q$ of state-of-the-art SiN microresonators ${ }^{48}$. Simulated DOPO convergence for $\mathbf{b} Q_{\text {int }}=1.26 \times 10^{6}$, which corresponds to a cavity lifetime $\tau_{\mathrm{p}}=0.52 \mathrm{~ns}$, and $\mathbf{c} Q_{\text {int }}=10 \times 10^{6}$, which corresponds to $\tau_{\mathrm{p}}=4.1 \mathrm{~ns}$, for critical coupling.

threshold pump powers. For a single DOPO in a microresonator with an intrinsic $Q$ of 1.26 million (our experiment), we observe a convergence time of $174 \mathrm{~ns}$ with a cavity lifetime of $0.52 \mathrm{~ns}$ based on our numerical model. For a microresonator with an intrinsic $Q$ of 10 million, we observe convergence times of $540 \mathrm{~ns}$. Experimentally in our 2-DOPO system, we achieve a convergence time $<310 \mathrm{~ns}$ with a computing rate of $400 \mathrm{kHz}$. Furthermore, we have performed numerical modeling of a $N=100$ DOPO system 
and observe an annealing time $T_{\text {ann }}=0.2 \mu$ s with a $35 \%$ groundstate success probability (see Supplementary Note 7 ). We calculate a time to solution, $T_{\text {sol }}=\frac{\log (0.01)}{\log (1-\mathrm{P})}=2.1 \mu \mathrm{s}^{6,23}$. As a comparison, the fiber-based DOPO system has a $T_{\text {sol }}=3.3 \mathrm{~ms}$ for solving cubic MAX-CUT problems and $T_{\text {sol }}=2.3 \mathrm{~ms}$ for dense MAX-CUT with $N=100^{23}$, suggesting that the spatialmultiplexing approach offers promise for accelerating the computing time of an Ising model. As the number of DOPOs in the network increases, the anneal time that corresponds to the pump turn-on time must be controlled to slow down the DOPO dynamics to prevent freeze-out effects that prevent the system from reaching the ground-state solution ${ }^{23,35}$. More specifically for our system of microresonator-based DOPOs, it has been reported elsewhere that the rate at which the DOPOs are tuned into resonance is a decisive parameter that determines the success probability of finding the ground-state solution (see Supplementary Note 8$)^{41}$. As the pump-to-resonance detuning dictates the power build-up within the microresonator, this observation is consistent with previous studies where the pump power was ramped up at a controlled rate to improve the performance of their systems ${ }^{20}$. In other words, the detuning is the more natural control parameter for our microresonator-based DOPOs, which must be carefully tuned. This fact will be subject to a more comprehensive investigation and reported elsewhere. We also observe that the oscillation threshold is reduced for a 2-DOPO system for both in-phase and out-of-phase couplings ${ }^{41}$, which leads to apparent faster convergence times as compared to a single DOPO. Investigations are ongoing for the optimal turn-on time as the problem size further increases for our spatialmultiplexed DOPO system. Finally, we have developed a preliminary 4-DOPO system and have observed DOPO generation in a single microresonator as shown in Supplementary Note 9. Future work will implement reconfigurable phase coupling between the DOPOs via integrated heaters.

\section{Discussion}

In conclusion, we demonstrate phase reconfigurable all-optical coupling between microresonator-based DOPOs in an integrated silicon nitride platform. We experimentally observe that the system is highly tolerant to the coupling phase between the DOPOs, offering flexibility in setting up the system when scaling to larger number of DOPOs. Since the system does not rely on time multiplexing, and the computation time is comparable to the build-up time of a single DOPO, it is possible to rapidly test the fidelity of the final state. To enable reconfigurable coupling between arbitrary DOPOs, a fraction of the DOPO power will be sent to a matrix of $2 \mathrm{~N} \times 2 \mathrm{~N}$ Mach-Zehnder interferometers (MZIs) to perform $N \times N$ arbitrary linear transformations ${ }^{49-51}$. To achieve this on a single device layer, intersections between waveguides are required. Our transmission measurements (see Supplementary Note 10) indicate $0.45 \mathrm{~dB}$ loss per intersection, which can be further optimized. In addition, we are investigating a multilayer design $^{52}$ where the coupling matrix based on MZIs is located in a different material plane, which uses silicon for the waveguides. Due to the larger thermo-optic effect in silicon, the MZIs can be further miniaturized such that each MZI element resides on a footprint of $100 \mu \mathrm{m} \times 100 \mu \mathrm{m}\left(0.01 \mathrm{~mm}^{2}\right)^{53}$, which is significantly smaller than that required for a SiN MZI $\left(0.7 \mathrm{~mm}^{2}\right)^{54}$. Such a design would enable all-to-all coupling among the DOPOs with arbitrary weightings and the resulting system should in principle allow the implementation of an arbitrary Hamiltonian. Our results provide the initial building blocks for the realization of a largescale DOPO network for studying nontrivial coupled oscillator dynamics, offering potential towards the realization of a chipbased photonic Ising machine.

\section{Methods}

Experimental setup. The setup is shown in Fig. 2a. Two tunable cw lasers (New Focus Velocity) are amplified using an erbium-doped fiber amplifier (EDFA) to use as pumps. The high-wavelength pump is modulated using an AOM, where the modulation depth and frequency are carefully chosen such that the DOPO signal reaches the noise level each time the AOM turned off. The pulse duration, repetition rate, and the extinction ratio are largely dependent on the quality factor of the microresonator ${ }^{39}$. For our system, with our pulse parameters we use an extinction ratio of 5:1 for our AOM. This extinction ratio is sufficient to reduce the pump power to a level below the oscillation threshold and, this together with our choice of pulse duration and repetition rate, allows for the DOPO signal to decay to a level below the noise before the initiation of the next period. In order to suppress the amplified spontaneous emission from the EDFA at the DOPO wavelength, we use 9-nm-wide bandpass filters after each EDFA. Fiber polarization controllers are used in each arm to set the input polarization to TE. For the chip output, the individual DOPO arms and the interferometer output are collected using a fiber collimator and the DOPO wavelength is filtered using a tunable bandpass filter with a $0.8-\mathrm{nm}$ bandwidth and sent to an InGaAs amplifier photodetector (150$\mathrm{MHz}$ bandwidth) and a 1-GHz real-time oscilloscope.

\section{Data availability}

The data that support the plots within this paper and other findings of this study are available from the corresponding author upon reasonable request.

\section{Code availability}

The modeling is described in the Supplementary information and the code is available from the corresponding author upon reasonable request.

Received: 6 March 2020; Accepted: 22 July 2020;

Published online: 17 August 2020

\section{References}

1. Backus, J. Can programming be liberated from the von Neumann style?: a functional style and its algebra of programs. Commun. ACM 21, 613 (1978)

2. Johnson, M. W. et al. Quantum annealing with manufactured spins. Nature 473, 194-198 (2011).

3. Bojnordi, M. N. \& Ipek, E. The memristive Boltzmann machines. IEEE Micro 37, 22-29 (2017).

4. Shin, J. H., Jeong, Y. J., Zidan, M. A., Wang, Q. \& Lu, W. D., Hardware acceleration of simulated annealing of spin glass by RRAM crossbar array. In 2018 IEEE International Electron Devices Meeting (IEDM), 3.3.1-3.3.4 (2018).

5. King, A. D., Bernoudy, W., King, J., Berkley, A. J., \& Lanting, T., Emulating the coherent Ising machine with a mean-field algorithm. Preprint at https://arxiv. org/abs/1806.08422 (2018).

6. Aramon, M. et al. Physics-inspired optimization for quadratic unconstrained problems using a digital annealer. Front. Phys. 7, 48 (2019).

7. Cai, F. et al. Power-efficient combinatorial optimization using intrinsic noise in memristor Hopfield neural networks. Nat. Electron. 3, 409-418 (2020).

8. Mahmoodi, M. R., Prezioso, M. \& Strukov, D. B. Versatile stochastic dot product circuits based on nonvolatile memories for high performance neurocomputing and neurooptimization. Nat. Commun. 10, 5113 (2019).

9. Yamamoto, Y., Takata, K. \& Utsunomiya, S. Quantum computing vs. coherent computing. N. Gener. Comput. 30, 327 (2012).

10. Utsunomiya, S., Takata, K. \& Yamamoto, Y. Mapping of Ising models onto injection-locked laser systems. Opt. Express 19, 18091 (2011).

11. Takata, K., Utsunomiya, S. \& Yamamoto, Y. Transient time of an Ising machine based on injection-locked laser network. N. J. Phys. 14, 013052 (2012).

12. Takata, K. \& Yamamoto, Y. Data search by a coherent Ising machine based on an injection-locked laser network with gradual pumping or coupling. Phys. Rev. A 89, 032319 (2014).

13. Utsunomiya, S. et al. Binary phase oscillation of two mutually coupled semiconductor lasers. Opt. Express 23, 6029 (2015).

14. Tradonsky, C. et al. Rapid laser solver for the phase retrieval problem. Sci. Adv. 5, eaax4530 (2019).

15. Babaeian, M. et al. A single shot coherent Ising machine based on a network of injection-locked multicore fiber lasers. Nat. Commun. 10, 3516 (2019).

16. Yamamoto, Y. et al. Coherent Ising machines-optical neural networks operating at the quantum limit. npj Quantum Inf. 3, 49 (2017).

17. Marandi, A., Wang, Z., Takata, K., Byer, R. L. \& Yamamoto, Y. Network of time-multiplexed optical parametric oscillators as a coherent Ising machine. Nat. Photonics 8, 937 (2014).

18. Inagaki, T. et al. Large-scale Ising spin network based on degenerate optical parametric oscillators. Nat. Photonics 10, 415 (2016). 
19. Wang, Z., Marandi, A., Wen, K., Byer, R. L. \& Yamamoto, Y. Coherent Ising machine based on degenerate optical parametric oscillators. Phys. Rev. A 88, 063853 (2013).

20. Inagaki, T. et al. A coherent Ising machine for 2000-node optimization problems. Science 354, 603 (2016).

21. McMahon, P. L. et al. A fully programmable 100-spin coherent Ising machine with all-to-all connections. Science 354, 614 (2016).

22. Guo, Y., Kroeze, R. M., Vaidya, V. D., Keeling, J. \& Lev, B. L. Sign-changing photon-mediated atom interactions in multimode cavity quantum electrodynamics. Phys. Rev. Lett. 122, 193601 (2019).

23. Hamerly, R. et al. Experimental investigation of performance differences between coherent Ising machines and a quantum annealer. Sci. Adv. 5, eaau0823 (2019).

24. Böhm, F., Verschaffelt, G. \& Van der Sande, G. A poor man's coherent Ising machine based on opto-electronic feedback systems for solving optimization problems. Nat. Commun. 10, 3538 (2019).

25. Prabhu, M. et al. Accelerating recurrent Ising machines in photonic integrated circuits. Optica 7, 551-558 (2020).

26. Roques-Carmes, C. et al. Heuristic recurrent algorithms for photonic Ising machines. Nat. Commun. 11, 249 (2020)

27. Pierangeli, D., Marcucci, G. \& Conti, C. Large-scale photonic Ising machine by spatial light modulation. Phys. Rev. Lett. 122, 213902 (2019).

28. Kumar, S., Zhang, H. \& Huang, Y.-P. Large-scale Ising emulation with four body interaction and all-to-all connections. Commun. Phys. 3, 108 (2020).

29. Tezak, N. et al. Integrated coherent Ising machines based on self-phase modulation in microring resonators. IEEE J. Sel. Top. Quantum Electron. 26 1-15 (2020).

30. Ising, E. Beitrag zur theorie des ferromagnetismus. Z. Phys. 31, 253 (1925).

31. Binder, K. \& Young, A. P. Spin glasses: experimental facts, theoretical concepts, and open questions. Rev. Mod. Phys. 58, 801 (1986).

32. Lucas, A. Ising formulations of many NP problems. Front. Phys. 2, 5 (2014).

33. Yamamura, A., Aihara, K. \& Yamamoto, Y. Quantum model for coherent Ising machines: discrete-time measurement feedback formulation. Phys. Rev. A 96, 053834 (2017).

34. Haribara, Y., Ishikawa, H., Utsunomiya, S., Aihara, K. \& Yamamoto, Y. Performance evaluation of coherent Ising machines against classical neural networks. Quantum Sci. Technol. 2, 044002 (2017).

35. Böhm, F. et al. Understanding dynamics of coherent Ising machines through simulation of large-scale 2D Ising models. Nat. Commun. 9, 5020 (2018).

36. Turitsyn, S. K., Bednyakova, A. E., Fedoruk, M. P., Papernyi, S. B. \& Clements, W. R. L. Inverse four-wave mixing and self-parametric amplification in optical fibre. Nat. Photonics 9, 608 (2015).

37. Okawachi, Y. et al. Dual-pumped degenerate Kerr oscillator in a silicon nitride microresonator. Opt. Lett. 40, 5267 (2015).

38. Takesue, H. \& Inagaki, T. $10 \mathrm{GHz}$ clock time-multiplexed degenerate optical parametric oscillators for a photonic Ising spin network. Opt. Lett. 41, 4273 (2016).

39. Okawachi, Y. et al. Quantum random number generator using a microresonator-based Kerr oscillator. Opt. Lett. 41, 4194 (2016).

40. Gaeta, A. L., Lipson, M. \& Kippenberg, T. J. Photonic-chip-based frequency combs. Nat. Photonics 13, 158 (2019).

41. Jang, J. K., Okawachi, Y. \& Gaeta, A. L. Dynamics of coupled microresonatorbased degenerate optical parametric oscillators. In Conference on Lasers and Electro-Optics, FTh1E.4 (Optical Society of America, 2018).

42. Luke, K., Dutt, A., Poitras, C. B. \& Lipson, M. Overcoming $\mathrm{Si}_{3} \mathrm{~N}_{4}$ film stress limitations for high quality factor ring resonators. Opt. Express 21, 22829-22833 (2013).

43. Soldano, L. B. et al. Planar monomode optical couplers based on multimode interference effects. J. Lightwave Technol. 10, 1843-1850 (1992).

44. Cunningham, J. E. et al. Highly-efficient thermally-tuned resonant optical filters. Opt. Express 18, 19055 (2010).

45. Joshi, C. et al. Thermally controlled comb generation and soliton modelocking in microresonators. Opt. Lett. 41, 2565 (2016).

46. Lugiato, L. A. \& Lefever, R. Spatial dissipative structures in passive optical systems. Phys. Rev. Lett. 58, 2209 (1987).

47. Dudley, J. M. \& Coen, S. Coherence properties of supercontinuum spectra generated in photonic crystal and tapered optical fibers. Opt. Lett. 27, 1180-1182 (2002).

48. Ji, X. et al. Ultra-low-loss on-chip resonators with sub-milliwatt parametric oscillation threshold. Optica 4, 619 (2017).
49. Reck, M., Zeilinger, A., Bernstein, H. J. \& Bertani, P. Experimental realization of any discrete unitary operator. Phys. Rev. Lett. 73, 58-61 (1994).

50. Clements, W. R., Humphreys, P. C., Metcalf, B. J., Kolthammer, W. S. \& Walmsley, I. A. Optimal design for universal multiport interferometers. Optica 3, 1460-1465 (2016).

51. Shen, Y. et al. Deep learning with coherent nanophotonic circuits. Nat. Photonics 11, 441 (2017).

52. MacFarlane, N., Kossey, M. R., Stroud, J. R., Foster, M. A. \& Foster, A. C. A multi-layer platform for low-loss nonlinear silicon photonics. APL Photonics 4, 110809 (2019).

53. Watts, M. R. et al. Adiabatic thermo-optic Mach-Zehnder switch. Opt. Lett. 38, 733-735 (2013).

54. Joo, J., Park, J. \& Kim, G. Cost-effective $2 \times 2$ silicon nitride Mach-Zehnder interferometric (MZI) thermo-optic switch. IEEE Photonics Technol. Lett. 30, 740-743 (2018).

\section{Acknowledgements}

This work was supported by Army Research Office (ARO) (grant W911NF-17-1-0016), National Science Foundation (NSF) (grant CCF-1640108), Semiconductor Research Corporation (SRC) (grant SRS 2016-EP-2693-A), and Air Force Office of Scientific Research (AFOSR) (grant FA9550-15-1-0303). This work was performed in part at the Cornell Nano-Scale Facility, which is a member of the National Nanotechnology Infrastructure Network, supported by the NSF, and at the CUNY Advanced Science Research Center NanoFabrication Facility. We also acknowledge useful discussions with A. Farsi, C. Joshi, C. Joshi, A. Mohanty, S. Ramelow, and L. Shao.

\section{Author contributions}

Y.O. and M.Y. designed the devices and experiment. Y.O., M.Y., and Y.Z. performed the experiments. J.K.J. performed numerical modeling. X.J. fabricated the devices. Y.O., M.Y., Y.Z., and B.Y.K. performed characterization of the DOPO devices. Y.O. and M.Y. analyzed the data. Y.O. prepared the manuscript in discussion with all authors. M.L. and A.L.G. supervised the project.

\section{Competing interests}

The authors declare no competing interests.

\section{Additional information}

Supplementary information is available for this paper at https://doi.org/10.1038/s41467020-17919-6.

Correspondence and requests for materials should be addressed to A.L.G.

Peer review information Nature Communications thanks the anonymous reviewer(s) for their contribution to the peer review of this work.

Reprints and permission information is available at http://www.nature.com/reprints

Publisher's note Springer Nature remains neutral with regard to jurisdictional claims in published maps and institutional affiliations.

Open Access This article is licensed under a Creative Commons Attribution 4.0 International License, which permits use, sharing, adaptation, distribution and reproduction in any medium or format, as long as you give appropriate credit to the original author(s) and the source, provide a link to the Creative Commons license, and indicate if changes were made. The images or other third party material in this article are included in the article's Creative Commons license, unless indicated otherwise in a credit line to the material. If material is not included in the article's Creative Commons license and your intended use is not permitted by statutory regulation or exceeds the permitted use, you will need to obtain permission directly from the copyright holder. To view a copy of this license, visit http://creativecommons.org/ licenses/by/4.0/.

(c) The Author(s) 2020 